\title{
Der Fall Libyen und die Norm der Schutzverantwortung
}

\section{Inhalt}

1. Einleitung

2. Die Genese der "responsibility to protect“" 136

3. Die Anwendung der ,responsibility to protect“ in Libyen 142

4. Fazit

\section{Einleitung}

Als am Abend des 19. September 1792 die Kanonade von Valmy nicht mit dem Sieg der Koalitionstruppen gegen das Heer des revolutionären Frankreich endete, notierte Johann Wolfgang von Goethe in seinem Tagebuch der Kampagne in Frankreich, die er für den Herzog von Weimar begleitete, einen geschichtsträchtigen Satz. Im Kreis der von Resignation und Erschöpfung gezeichneten Soldaten habe er (offensichtlich nicht ohne eine gewisse Ironie) gesagt: „Von hier und heute geht eine neue Epoche der Weltgeschichte aus, und ihr könnt sagen, ihr seid dabei gewesen." (Goethe 1988: 411) Wenn auch die Umstände deutlich andere sind, so scheint die Resolution des Sicherheitsrates der Vereinten Nationen zur Situation in Libyen schon kurz nach ihrer Verabschiedung ein ähnlich herausragendes Gefühl der Zeitzeugenschaft „von denen, die dabei gewesen sind“, hervorgebracht zu haben. Der UNO-Generalsekretär nannte die Resolution unmittelbar nach der Abstimmung am Abend des 17. März 2011 eine „,historic decision“ (Ban 2011), im bedrängten Bengasi jubelten die Aufständischen und einige Wochen später nannten die Staats- und Regierungschefs der USA, Großbritanniens und Frankreichs die Resolution ebenfalls „historic“ (Obama/Cameron/Sarkozy 2011) - nicht zuletzt, weil sie ein ,unprecedented international legal mandate" beinhalte: Den Einsatz militärischer Mittel zum Schutz der Bevölkerung eines Staates, dessen Regierung sich gegen seine Einwohner gewandt hatte.

Tatsächlich hat der Sicherheitsrat in der Resolution 1973 (UN Doc. SR/Res 1973 vom 17.3.2011) in einem qualitativen neuen Sinn eine Norm bekräftigt, die seit dem Ende der 1990er Jahre unter dem Schlagwort „responsibility to protect“ bzw. dem 
Kürzel R2P diskutiert wird (vgl. zum Überblick u.a. Fröhlich 2006; Strauss 2009; Verlage 2009; Bellamy 2009; Evans 2009). Mit dieser Norm ${ }^{1}$ sind eine Reihe von Erfahrungen und Kontroversen verbunden, die im Spannungsfeld von internationaler Intervention und nationaler Souveränität stehen. R2P zielt auf nichts weniger als auf eine Neuakzentuierung von ethischen Maßstäben in der internationalen Politik. Vor diesem Hintergrund ist die Bekräftigung dieser Norm, die in relativ kurzer Zeit Wirkmächtigkeit erlangt hat, ein Lehrstück für den Normwandel in den internationalen Beziehungen. Gleichzeitig sind mit dem Konzept neben den Neuerungen auch eine Reihe von Problemen verbunden, die gerade in Bezug auf Libyen deutlich zutage treten. Vor diesem Hintergrund will der Beitrag zunächst die „Genese“ dieser Norm kurz skizzieren, ihre Anwendung am Fall Libyens diskutieren und schließlich nach den konzeptionellen wie praktisch-politischen Konsequenzen der Resolution 1973 fragen.

\section{Die Genese der ,responsibility to protect“"}

Der völkerrechtlich legitime Einsatz von Gewalt wird in der Charta der Vereinten Nationen durch wenige Artikel definiert: Artikel 2 Ziffer 4 fordert die Staaten auf, ,jede gegen die territoriale Unversehrtheit oder die politische Unabhängigkeit eines Staates gerichtete oder sonst mit den Zielen der Vereinten Nationen unvereinbare Androhung oder Anwendung von Gewalt" zu unterlassen. Dies war ja gerade eine Lehre der internationalen Politik über Jahrhunderte hinweg gewesen: Die Aggression, der Überfall auf kleinere, benachbarte oder als konkurrierend wahrgenommene Länder war die Kriegsursache schlechthin - wie immer auch die einzelnen Regierungen ihren Waffengang vorgeblich oder tatsächlich legitimiert sahen. Artikel 2.4 formuliert deshalb ein allgemeines Gewaltverbot. Dieses erhält in Artikel 2 Ziffer 7 sozusagen noch eine Bestärkung, indem die Charta keine Möglichkeiten „zum Eingreifen in Angelegenheiten, die ihrem Wesen nach zur inneren Zuständigkeit

1 Die Etikettierung der R2P als „Norm“ ist durchaus umstritten. Luck, der Finnemore und Sikkink in der Definition einer Norm als ,,a standard of appropriate behaviour for actors with a given identity“ (1998: 891) im akademischen Sinne folgt, vermeidet den Begriff in seiner politischen Arbeit als Sonderberater des UN-Generalsekretärs, da „Norm“ auf internationaler Ebene zu sehr mit der Assoziation einer völkerrechtlich bindenden Qualität verbunden sei (Luck 2010: 109). In ähnlichem Sinne akzentuiert Bellamy die Begrifflichkeiten „Konzept“ und „Prinzip“ anstelle der Norm (2009: 4-7). Die in diesem Beitrag skizzierte Nähe der R2P zur wissenschaftlichen Debatte um Normenwandel rechtfertigt jedoch die Nutzung des Begriffs. Zugleich verwendet der Beitrag angesichts der Herausbildung dieser Norm über verschiedene Etappen hinweg durchgängig die Abkürzung R2P und unterscheidet nicht zwischen R2P (etwas als Kürzel des ICISS-Vorschlags) und einer davon zu trennenden RtoP (ein Kürzel, das Luck für die spezifische Zugangsweise der Vereinten Nationen verwendet (2010: 124, FN 1)). 
eines Staates gehören“, eröffnet. Hier wird, wenn auch nicht dem Worte nach, unmissverständlich die Souveränität der Staaten geschützt. Die Wahrung der Eigenständigkeit und das Beharren auf Nichteinmischung in innere Angelegenheiten hatten sich bereits im Westfälischen Frieden von 1648 als Friedensformel erwiesen.

Die Ausnahme von diesem Grundsatz findet sich jedoch gemäß der Logik eines Systems kollektiver Sicherheit in den möglichen Zwangsmaßnahmen („Waffengewalt [...] im gemeinsamen Interesse“, Präambel der Charta), die der Sicherheitsrat der Vereinten Nationen im Falle von Bedrohungen der internationalen Sicherheit und des Weltfriedens anordnen kann (Kapitel VII). An dieser Stelle muss nicht nochmals darauf hingewiesen werden, dass das damit etablierte System kollektiver Sicherheit (zu dem auch noch die Möglichkeit der unmittelbaren Selbstverteidigung nach Art. 51 zählt) im System der Vereinten Nationen durch politische Divergenzen lange Zeit allenfalls defizitär funktionierte. Eine Konsequenz aus diesem Umstand war ja gerade die „Erfindung“ der in der Charta nicht explizit vorgesehenen Friedenstruppen der UNO. Wichtiger für das Verständnis der Debatte zur Schutzverantwortung ist allerdings der Umstand, dass sich die Konfliktstruktur im internationalen System stark gewandelt hat: Die meisten Konflikte sind nicht mehr zwischen-, sondern innerstattlicher Natur (Human Security Center 2005). Und in diesen Konflikten stellt sich die vormalige Friedensformel der Souveränität auch als Kriegsgrund dar (Fröhlich 2004 a): Regierungen, die Gewaltmittel gegen die eigene Bevölkerung einsetzen, nutzten den Verweis auf die Souveränität als Abwehrschild gegenüber Kritik und Einmischung aus dem Ausland.

Die 1990er Jahre stehen dabei für mehrere schmerzliche Beispiele eines solchen Vorgehens. Ca. 800.000 Tote des Völkermords in Ruanda und 8.000 Tote des Massakers von Srebrenica stellten die Debatte um das Verhältnis von Souveränität und Intervention auf eine neue Grundlage. In beiden Fällen fehlte es der internationalen Gemeinschaft an Handlungsmöglichkeiten und an Handlungswillen, effektiv gegen solche Massentötungen vorzugehen. Diese Problematik fand 1999 in der vom Sicherheitsrat nicht autorisierten Intervention der NATO im Kosovo-Konflikt einen weiteren Ausdruck: Hier wurde gehandelt - allerdings unter Umgehung des Systems kollektiver Sicherheit. Vor diesem Hintergrund wurde auf Anregung des damaligen UN-Generalsekretärs Kofi Annan und der kanadischen Regierung eine Expertenkommission eingesetzt, die das schwierige Spannungsfeld zwischen Souveränität und Intervention neu vermessen sollte: Die International Commission on Intervention and State Sovereignty (ICISS). Deren Bericht bringt zum ersten Mal das Konzept der R2P prominent hervor.

Die zentrale These dieses Berichts stellt den Schutz der Menschen in den Mittelpunkt staatlicher wie internationaler Politik (ICISS 2001; vgl. Fröhlich 2006). Der 
Schutz der Menschen wird zur konstitutiven Bedingung von Souveränität, da er (neben jüngeren Entwicklungen im Völkerrecht und Menschenrechtsschutz) von Beginn an zu den ,obligations inherent in the concept of sovereignty” (ICISS 2001: xi) gehört. Tatsächlich lässt sich der konstitutive Schutzgedanke ideengeschichtlich bis an den Ursprung des Souveränitätsbegriffs verfolgen (Fröhlich 2005). Eine Lesart von Souveränität als Abwehrschild, hinter dem Machthaber grobes Unrecht an ihren Bürgern verüben dürfen, war insofern nie authentisch - wenngleich sie oft genug und in mancherlei Variation in der internationalen Politik bemüht wurde. Ausgehend von dieser Bestimmung staatlicher Souveränität formuliert die ICISS: ,[T]he idea [is] that sovereign states have a responsibility to protect their own citizens from avoidable catastrophe - from mass murder and rape, from starvation but that when they are unwilling or unable to do so, that responsibility must be borne by the broader community of states." (ICISS 2001: viii) Wenn also die primäre Verantwortung eines Staates gegenüber seinen eigenen Bürgern nicht wahrgenommen wird, dann tritt die internationale Gemeinschaft in eine Ersatzfunktion: „Where a population is suffering serious harm, as a result of internal war, insurgency, repression or state failure, and the state in question is unwilling or unable to halt or avert it, the principle of non-intervention yields to the international responsibility to protect." (ICISS 2001: xi) Diese Aussage leitet in mehrfacher Hinsicht einen Paradigmenwechsel ein: Zum einen findet sich kein Verweis auf traditionelle Argumentationsmuster von einem (wie auch immer) nationalstaatlich reklamierten Recht auf Intervention in einen anderen Staat; die Rede ist vielmehr von einer Verantwortung der Staatengemeinschaft. Zum anderen wollen die Mitglieder der ICISS die Verantwortung zum Schutz ausdrücklich nicht nur als militärische Aufgabe verstanden wissen, sondern stellen der ,,responsibility to react" mittels militärischer Zwangsmaßnahmen die „responsibility to prevent“ und die „responsibility to rebuild“" an die Seite (ICISS 2001: xi). Während die „responsibility to react“ auch Sanktionen umfassen könne und militärische Interventionen nur ,in extreme cases“ erfolgen sollten, wird die präventive Verantwortung als "the single most important dimension of the responsibility to protect" bezeichnet. Präventive Maßnahmen, die auf ,both the root causes and direct causes of internal conflict and other man-made crises putting populations at risk" (ICISS 2001: xi) zielten, müssten zudem sämtlich ausgeschöpft sein, „before intervention is contemplated”.

Sollte es dennoch zum Abwägen militärischer Optionen kommen, schlägt der Kommissionsbericht eine Reihe von Prinzipien vor, welche die Entscheidung bestimmen sollten. Die ersten beiden Prinzipien behandeln die Schwelle, ab der militärische Interventionen überhaupt in Frage kommen. Genannt werden unter dem Stichwort ,just cause”: „A. large scale loss of life, actual or apprehended, with 
genocidal intent or not, which is the product either of deliberate state action, or state neglect or inability to act, or a failed state situation; or B. large scale 'ethnic cleansing', actual or apprehended, whether carried out by killing, forced expulsion, acts of terror or rape." (ICISS 2001: xii) Die ICISS bindet den Einsatz von Gewalt darüber hinaus noch an ,precautionary principles“, die eine mögliche Eingriffsentscheidung an die Abwägung der Prinzipien von „right intention“, „last resort“, „proportional means“ und „reasonable prospects“ verpflichtet (ebd.). Gerade in der knappen Erläuterung dieser Prinzipien wird beständig auf die Perspektive der Opfer verwiesen, die für die Prüfung der Prinzipien entscheidend sei.

Bei näherem Hinsehen erweisen sich die Prinzipien, zu denen noch einige operative Forderungen wie etwa ein klares Mandat kommen, zutiefst verankert in der Denkfigur des ,gerechten Krieges“ und seinen Kategorien von causa iusta, recta intentio und legitima auctoritas (so auch Fixdal/Smith 1998; vgl. auch Chesterman 2001). Mehr noch: Dieselben Schwierigkeiten und konzeptionellen Probleme, denen die Lehre vom gerechten Krieg seit dem Mittelalter ausgesetzt ist, finden sich auch bei den Prinzipien der ICISS. Da ist zunächst der Umstand, dass die Prinzipien zugleich gewaltlegitimierende wie gewaltlimitierende Argumentationen formulieren. Zweitens mögen die Kriterien in ihrer allgemeinen Form sinnvoll erscheinen das Problem ist jedoch, dass sie ihrerseits Begriffe enthalten, über deren politische, völkerrechtliche und ethische Einschätzung trefflich gestritten werden kann (,letztes“ Mittel, „Ernsthaftigkeit“ der Bedrohung etc.). Der zentrale Unterschied zwischen der klassischen Lehre vom gerechten Krieg und den Vorschlägen der ICISS besteht allerdings (abseits der Veränderung von Zerstörungspotentialen etc.) in dem Umstand, dass die Entscheidung über die Auslegung und Anwendung der Prinzipien nicht einem Herrscher oder Staat, sondern einem multilateralen Gremium, dem UNSicherheitsrat, übertragen wird. Der Bericht formuliert unter dem Stichwort "right authority", eine Autorisierung durch den Sicherheitsrat ,should in all cases be sought prior to any military intervention" (ICISS 2001: xii). In dem Wörtchen „should" findet sich jedoch eine Tür, die die monopolartige Stellung des Sicherheitsrates etwas relativiert. Ihm wird nämlich implizit auch eine Art von ,responsibility" zugeschrieben, die mit seiner einzigartigen rechtlichen Stellung einhergeht: So müsse der Rat sich umgehend um Krisensituationen kümmern und aussagefähige Verifikationsmechanismen etablieren. Zudem sollten sich die ständigen Mitglieder im Rat bei Fragen der Schutzverantwortung mit ihrem Veto-Recht zurückhalten (ICISS 2001: xiii) - eine politisch äußerst brisante Forderung, die sich nicht auf die bisherige Praxis der ,permanent five“ (P5), das heißt der fünf ständigen Mitglieder des UN-Sicherheitsrates, stützen kann. 
Von Beginn an formierte sich gegenüber der „responsibility to protect“ die Kritik an einer möglicherweise souveränitätsaushöhlenden Auslegung, die als Deckblatt für Machtprojektionen alten Stils herhalten könnte. Gerade der Irak-Krieg 2003 und die (beispielsweise durch den britischen Premierminister Tony Blair hergestellte) Assoziation dieses Waffengangs mit der R2P erhöhten diesen Verdacht und die Zurückhaltung vieler Mitgliedstaaten der Vereinten Nationen. Vor diesem Hintergrund ist es erstaunlich, dass diese das Konzept dann doch in das Abschlussdokument des Weltgipfels 2005 aufnahmen. Tatsächlich konnte diese einhellige Aufnahme in eine (zunächst nicht rechtsverbindliche) Resolution der Generalversammlung nur auf dem Wege einer deutlichen Reduzierung der Substanz des Konzepts erreicht werden. Das Abschlussdokument erwähnt gerade nicht all jene Einzelforderungen der ICISS, sondern stellt die R2P im Wesentlichen als Bekräftigung von bereits geltendem Völkerrecht dar und bindet sie zugleich nochmals deutlich an die Tatbestände ,genocide, war crimes, ethnic cleansing and crimes against humanity“ (UN Doc. A/RES/60/1 vom 24.10.2005, para. 138-140). Wie umstritten das Konzept blieb, zeigt auch der Umstand, dass die Stelle eines Sonderberaters, der den Generalsekretär zur „responsibility to protect“ beraten sollte, nicht einmal im offiziellen Titel die Formel der R2P tragen durfte.

Trotz dieser Vorbehalte wurde die R2P seit dem 2005er Gipfeldokument in einer ganzen Reihe von Krisen- und Konfliktsituationen diskutiert. Dabei ging die Initiative nicht selten auf zivilgesellschaftliche Akteure zurück: Die Krise in Darfur, die Gewaltausbrüche nach der Präsidentschaftswahl in Kenia, die Verhinderung humanitärer Hilfe nach dem Zyklon Nargis in Myanmar oder die Situation nach den Unruhen in Kirgisistan wurden in das Licht der R2P gerückt (vgl. etwa Strauss 2009; Bellamy 2009; Kreuter-Kirchhof 2010). Das Konzept wurde jedoch nicht offiziell, das heißt auf der Ebene zwischenstaatlicher Bestätigung oder Anwendung, zum Einsatz gebracht. Die Mitglieder des UN-Sicherheitsrates konnten sich im Laufe der folgenden Jahre aufgrund der weiterhin bestehenden Zurückhaltung gegenüber dem Konzept kaum zu einer offiziellen Bezugnahme auf die R2P einigen. Vor der Libyen-Krise geschah dies lediglich als sprachliche Übernahme oder Variation des Beschlusses von 2005 in thematischen Resolutionen zum Schutz von Zivilisten in bewaffneten Konflikten (etwa UN Doc. SR/Res 1674 vom 28.4.2006) oder bei Resolutionen zu bestehenden Missionen (etwa UN Doc. SR/Res 1949 vom 23.11.2010). Doch selbst die formal anmutende Aufnahme einer solchen Bekräftigung in thematischen Resolutionen war durchaus umstritten (Bellamy 2009: 133-139). Dies lag an gleich mehreren Faktoren: Zum einen sind im Rat (gerade auch bei den Ständigen Mitgliedern) durchaus Stimmen vertreten, die keinen Präzedenzfall zur Einmischung von außen schaffen wollen, der dann auf innenpoliti- 
sche Problemfälle im eigenen Land übertragen werden könnte. Zum anderen tut sich der Rat grundsätzlich schwer damit, sich in seinen Handlungen über Einzelfallentscheidungen hinaus grundsätzlich zu binden.

Immerhin: Wenn auch die „responsibility to protect“ als Konzept kaum direkte Erwähnung fand, so hat sich der Sache nach dieser Gedanke (aufbauend auf dem humanitären Völkerrecht) als Bestandteil des Auftrags diverser Friedenstruppen Bahn gebrochen. Der Textbaustein ,to protect civilians under imminent threat of physical violence" findet sich mit Variationen in den Mandaten der Missionen in Burundi, der Elfenbeinküste, Darfur, der Demokratischen Republik Kongo, Haiti, Liberia und Sierra Leone (vgl. Fröhlich 2004 b; Holt/Berkman 2006). Diese Bemühungen zum Schutz von Zivilisten (,protection of civilians“, POC) sind zwar verwandt zur R2P, gehen jedoch viel spezifischer auf die Reformdebatte der Friedensoperationen zurück und haben als solche auch ihre eigene Entwicklungsgeschichte in der Praxis des Rates. Tatsächlich hatte das Gipfeldokument von 2005 ja auch zunächst eine weitere Debatte des Themas in der Generalversammlung angemahnt (para. 139). Auch dieser Weg erwies sich als sehr mühsam. Aufbauend auf einem Bericht des Generalsekretärs (UN Doc. A/63/677 vom 12.1.2009), der eine auf drei Pfeilern ruhende Strategie zur Umsetzung der R2P skizzierte, ${ }^{2}$ konnte sich die Vollversammlung nur auf eine Resolution einigen, die aus ein paar dürren Zeilen bestand, die die R2P von 2005 mit dem Wort „recall“ anstelle von „reaffirm“ nennt und ansonsten beschließt, die Diskussion weiter fortzuführen (UN Doc. A/Res/ 63/308 vom 7.10.2009). Auch vor diesem Hintergrund ist die Bezugnahme auf und sogar Durchsetzung der R2P mittels einer Kapitel VII-Resolution durch den Sicherheitsrat angesichts der Situation in Libyen erstaunlich und markiert eine besondere Etappe in der Herausbildung dieser Norm. ${ }^{3}$

Tatsächlich lässt sich die Herausbildung der R2P bemerkenswert gut in den Kategorien des Lebenszyklus von Normen erfassen, den Finnemore und Sikkink be-

2 Die Pfeiler umfassten in einer merklich auf die Bedenken der Mitgliedstaaten eingehenden Diktion 1. ,the protection responsibilities of the State“, 2. ,international assistance and capacity building" sowie 3. ,timely and decisive response". R2P wurde in klaren Worten als "an ally of sovereignty, not an adversary" (UN Doc. A/63/677 vom 12.1.2009, para. 10) beschrieben; das hier vorgelegte Konzept sei „narrow and deep“. Edward Luck, der den Bericht vorbereitet hat, verteidigte diese Vorgehensweise im Sinne einer Wahrung des sehr fragilen Konsenses von 2005: ,It was critical at that early stage of R2P development [...] to avoid falling into the UN's usual trap of making straightforward concepts incoherent, unintelligible, and unusable by stretching them to cover more and more issues and concerns. The secretary-general wanted to make R2P operational, not just appealing, and this required discipline, constraint, and consistency in its application." (Luck 2010: 115)

3 Vor der Resolution 1973 hatte bereits UN Doc. SR/Res. 1970 vom 26.2.2011 "the Libyan authorities' responsibility to protect its population" angemahnt und spezifische Sanktionen nach Art. 41 verhängt. 
schrieben haben (Finnemore/Sikkink 1998; so auch bezeichnenderweise Luck 2010). Danach wären die Initiativen der ICISS und des UN-Generalsekretärs Kofi Annan, der hier als einer der entscheidenden ,norm entrepreneurs“ (Finnemore/ Sikkink 1998: 896-899) auftritt, Teil der ersten Phase des Zyklus, der ,norm emergence“. Diese wird von einem ,tipping point" abgeschlossen, an den sich eine „,norm cascade" anschließt: Nachdem eine kritische Anzahl von Staaten die Norm anerkannt haben, wird sie mehr und mehr zum Bezugspunkt der Debatte und erreicht im Laufe der Zeit und über wiederholte Bekräftigung und Anwendung die dritte Phase des Normzyklus, die ,internalization“, in der die Norm sozusagen unhinterfragt als Standard angemessenen Verhaltens gilt. Die Aufnahme der R2P in das einstimmig von der bis dahin größten Zusammenkunft von Staats- und Regierungschefs beschlossene Gipfeldokument 2005 kann in einer solchen Lesart als Entsprechung des ,tipping point“" gelten. Allerdings stellt sich die Realität der R2P an dieser Stelle etwas quer zu den allgemeinen Annahmen des Normenzyklus: Zum einen ist das Gipfeldokument ,mehr“ als der geforderte ,tipping point“ - nicht nur eine kritische Masse von Staaten, sondern praktisch alle Staaten nahmen die R2P an. Zum anderen ist diese Annahme zugleich ,weniger" als das, was Finnemore und Sikkink unter dem ,tipping point“ verstehen - die Erklärung der Generalversammlung ist nicht nur völkerrechtlich unverbindlich, sondern hat ihrerseits den Inhalt der R2P stark verändert und eingeschränkt. Selbst dieser Inhalt war jedoch weiterhin umstritten. Dass es trotzdem im Jahr 2011, zehn Jahre nach der Formulierung der Norm, zu ihrer ersten Anwendung kam, hängt mit der Besonderheit der Krise in Libyen zusammen.

\section{Die Anwendung der „,responsibility to protect" in Libyen}

Auch wenn die R2P aus dem Abschlussdokument von 2005 gerade nicht all jene Kriterien und Kategorien der ICISS aufnimmt, so erweist sich deren Güte darin, dass sie als gute Strukturierung der Entscheidungssituation in Libyen gelten kann. Bezüglich des ,just cause“ erfüllte die Lage in Libyen sicherlich das Schwellenkriterium ,large scale loss of life, actual or apprehended, with genocidal intent or not, which is the product either of deliberate state action, or state neglect or inability to act, or a failed state situation" (ICISS 2001: xii). Anfang März 2011 gingen internationale Organisationen und Nichtregierungsorganisationen bereits von mehreren Tausend Toten aus (vgl. Müller 2011). Der Angriff von bevölkerten Städten (Bengasis Einwohnerschaft liegt bei über 600.000 Menschen) hätte diese Opferzahlen deutlich in die Höhe treiben können. Zudem stand eine in vielen anderen Konflikten schwer zu beantwortende Frage hier außer Zweifel: Muammar al-Gaddafi und sein 
Sohn Saif al-Islam hatten explizit vor laufenden Kameras und Mikrofonen mit Vergeltungsaktionen und Massakern gedroht (vgl. The Editors 2011; Euronews 2011) und erfüllten damit auch das Kriterium ,national authorities manifestly fail to protect" aus dem Abschlussdokument von 2005 (para. 13). Damit war die Situation in Libyen bei aller Ambivalenz der militärischen Lage erstaunlich eindeutig: Hier war (anders als im Irak-Krieg 2003) eine Mehrheit des Rates handlungsbereit, hier hatte man (anders als bei Omar al-Bashir in Darfur) einen Diktator, der expressis verbis Massaker an der Zivilbevölkerung ankündigte. Sogar die libysche UN-Delegation in New York hatte sich (anders als im Falle Ruandas 1994) von der eigenen Regierung losgesagt und zum Handeln aufgerufen. Selbst die - auch von Deutschland aufgestellten - Hürden unterstützender Voten der Arabischen Liga, der Organisation der islamischen Konferenz und des Golf-Kooperationsrates wurden genommen. Sicher gilt auch, dass das Votum der Arabischen Liga mit Zweideutigkeiten besetzt ist und sich die wirkliche militärische Beteiligung arabischer Staaten an der Operation rasch vermindert hat. Und dennoch: Die Entscheidung des Sicherheitsrates markiert eine Art weiteren ,tipping point“ in der Genese der R2P. Sicher ist die Operation „Odyssey Dawn“"schon zu Beginn in mannigfaltige Schwierigkeiten geraten, die im Übrigen auch an die Erfahrungen im Kosovo erinnern (O'Hanlon 2011; Mueller 2011). Der Sonderberater des UN-Generalsekretärs, Edward Luck, mahnt jedoch aus Sicht der R2P deutlich, den völkerrechtlich legitimierten Auftrag zum unmittelbaren Schutz der Zivilbevölkerung nicht durch politische Differenzen über das militärische Vorgehen zu beschädigen (FAZ 2011).

Der Text der Resolution 1973 weist jedenfalls ein beachtliches Handlungsspektrum auf: Sie verlangt einen Waffenstillstand und ein Ende der Angriffe auf Zivilisten (para. 1), unterstützt die Entsendung eines UN-Gesandten, um politische Optionen zur Lösung der Krise auszuloten, die die Forderungen des libyschen Volkes aufnehmen (para. 2), und fordert die libysche Führung auf, ,all measures to protect civilians" zu ergreifen. Hierin findet sich zum einen ein gestaffeltes Spektrum an Tätigkeiten, das sowohl die Dualität von staatlicher und internationaler Verantwortlichkeit der R2P ernst nimmt sowie zugleich die Trias von prevent, react und rebuild andeutet. Absatz 4 der Resolution bietet gleich mehrere, sehr außergewöhnliche Punkte: Zum einen werden einzelne Mitgliedstaaten oder Regionalorganisationen nach Meldung beim Generalsekretär autorisiert ,to take all necessary measures $[\ldots]$ to protect civilians and civilian populated areas under attack". Hier findet sich mit dem Hinweis auf alle notwendigen Maßnahmen die UN-Chiffre für den Einsatz von militärischer Gewalt. Dass diese nicht an eine im Voraus spezifizierte Staatengruppe übertragen wird, sondern nach dem Muster einer losen „Koalition der Willigen", ist sehr selten. In diesem Absatz findet sich zugleich der Ausschluss 
ausländischer Besatzungstruppen - eine Formulierung, die eine Präsenz ähnlich wie im Irak untersagt, andererseits aber auch den Einsatz von zeitlich befristeten Spezialeinheiten durchaus erlaubt.

Absatz 4 der Resolution 1973 steht neben und zusätzlich zur Etablierung einer Flugverbotszone, die dann in den Absätzen 6 bis 8 geregelt wird. Hier findet sich wieder der Hinweis auf ,all necessary measures“. Im Weiteren bekräftigt die Resolution die Überweisung des Falles Libyen an den Internationalen Strafgerichtshof sowie die gezielten Sanktionen gegen das libysche Führungspersonal. Auffällig und fast schon in den Bereich der ,rebuild“-Kapazitäten deutend - ist zudem Absatz 20, der die Absicht bekundet, die im Rahmen der finanziellen Sanktionen eingefrorenen Auslandsguthaben der libyschen Führungsriege baldmöglichst „for the benefit of the people of the Libyan Arab Jamahiriya" bereitzustellen (para. 20). Hier wird also eine ganze Klaviatur unterschiedlicher Maßnahmen zum Einsatz gebracht, die in ihrer Unterschiedlichkeit durchaus das Spektrum des R2P-Ansatzes abbildet. Der explizite Bezug zu R2P findet sich in den präambulatorischen Absätzen. Hier wird einerseits auf die ,responsibility of the Libyan authorities to protect the Libyan population" und zugleich auf die Verantwortung aller Konfliktparteien zum Schutz von Zivilisten verwiesen - eine deutliche Inpflichtnahme auch der Aufständigen.

Die Umsetzung der militärischen Operation dauert zum Zeitpunkt des Verfassens dieses Beitrags an. Unabhängig vom Ausgang der Operation lassen sich jedoch schon jetzt einige konzeptionelle und praktisch-politische Konsequenzen aus dem Fall Libyen und der Norm der Schutzverantwortung ableiten.

\section{Fazit}

Die rasche Etablierung der ,responsibility to protect“ und die fortbestehenden Meinungsverschiedenheiten über die Legitimation des Einsatzes und die Auslegung des Mandats in Libyen (vgl. etwa einerseits Bennis 2011 und andererseits Sinjen 2011) zeigen eine Art Jetlag-Effekt: Der Problemdruck hat die Norm auffallend schnell herausgebildet, ohne dass damit schon die jeweilige Antwort auf politisch vertrackte, rechtlich und ethisch ambivalente sowie militärisch riskante Herausforderungen gegeben wäre. Das Versprechen von R2P liegt nach Bellamy denn auch nicht in der scheinbaren Auflösung dieser Widersprüche und Schwierigkeiten eines militärischen Einsatzes, sondern darin, "the frequency of all-or-nothing decisions" (2009: 4) zu vermindern. Die Neuakzentuierung von Verantwortlichkeiten, die Betonung der Perspektive der Opfer und der Versuch, Legalität und Legitimität von Handlungen der internationalen Gemeinschaft in Krisensituationen neu auszubalancieren (Doyle 2011), sollen zusammengenommen über die Zeit wirken. In diesem Kontext 
sei daran erinnert, dass die ICISS ihre Rolle ähnlich verstand wie die BrundtlandKommission: So wie damals der Gegensatz von wirtschaftlicher Entwicklung und Umweltschutz in das integrative Leitbild der nachhaltigen Entwicklung überführt wurde, sollte R2P den Gegensatz zwischen Souveränität und Intervention versöhnen (ICISS 2001: 81). Die Beachtung und Umsetzung von Nachhaltigkeit bleibt eine ständig neu zu lösende Aufgabe der internationalen Politik. Für die Beachtung und Umsetzung der R2P gilt dies ebenso. In dieser langfristigen Perspektive bietet auch das Beispiel der R2P einige konzeptionelle Konsequenzen für das Verständnis von Normwandel in den internationalen Beziehungen. So konnte im Großen und Ganzen das Modell von Finnemore und Sikkink auf die Herausbildung der „responsibility to protect" angewandt werden. Mehr noch: Finnemore und Sikkink verweisen auf frühere Arbeiten, die feststellen, dass Normen, die sich auf die Vermeidung körperlichen Schadens, die Übernahme einer Opferperspektive und Gleichheitsvorstellungen beziehen, besonders effektiv Wirkungen über Kulturen und Gesellschaften hinweg erzielen können (Finnemore/Sikkink 1998: 907). Der Blick auf R2P scheint diese Erkenntnis klar zu unterstützen. Hierin mag eine weitere Erklärung für die relative Schnelligkeit der Etablierung der Norm liegen. Verbunden mit dieser Einsicht ist im Übrigen der Hinweis auf die normbefördernde Möglichkeit, ,graphic images of bodily harm“ (908) einsetzen zu können. Auf diesem Wege bekommt die intensive Nutzung von neuen Kommunikationstechnologien (wie sie gerade den ,arabischen Frühling“ kennzeichnet) einen konzeptionell abgesicherten Ort innerhalb der begünstigenden Faktoren eines Normwandels.

Der primär konstruktivistische Blick auf die Entwicklung von Normen, den Finnemore und Sikkink ausdrücklich mit Überlegungen der Rational choice verbinden wollen, verweist daneben auf eine Mischung aus normgeleiteten und interessengeleiteten Motivlagen, wobei ja gerade die Neuformulierung von Interessen den Schnittpunkt zwischen rationalen und konstruktivistischen Annahmen darstellt. Auch hier passt das Beispiel der R2P: Schon die ICISS hatte in ihrem Bericht deutlich anerkannt, dass politische Akteure nie nur aus normativen Gesichtspunkten handeln würden (2001: xii). Natürlich verdrängen normative Beweggründe nicht einfach vorhandene wirtschaftliche, geostrategische oder andere „materielle“ Interessen. Dieser praktisch-politische Zusatz verbindet sich (etwa mit Blick auf die zentrale Rolle der französischen Regierung bei der Ausarbeitung der Resolution) mit der theoretisch-konzeptionellen Annahme von Finnemore und Sikkink, dass „the desire of state leaders to enhance their self-esteem facilitate norm cascades“ (895). Gleichzeitig gilt aber auch, wie nicht zuletzt die außergewöhnliche Enthaltung des langjährigen R2P-Unterstützers Deutschland bei der Libyen-Abstimmung zeigt, dass selbst in einem solch herausgehobenen Moment der Normentwicklung 
einzelne Akteure offenbar noch zu unvorhergesehenen Positionswechseln fähig sind. ${ }^{4}$ Der Blick auf die R2P sollte nicht zu einer Verkürzung der Analyse des Geschehens beitragen. Festzuhalten ist jedoch, dass die Bezugnahme auf diese Norm notwendig zum Verständnis der Konfliktsituation und der Lösungsversuche in Libyen ist.

Luck jedenfalls bilanziert eine auffallende Übereinstimmung zwischen seinen Erfahrungen mit der R2P und dem Modell des Lebenszyklus von Normen (Luck 2010: 110). Zugleich schränkt er ein: „There have been more stops, starts, detours, and regeneration in R2P's young life than any chart could properly depict" (ebd.) und folgert: "[T] he model fails to incorporate the interactive and sometimes even dysfunctional nature of international politics, assuming an overly linear conception of progress." (Luck 2010: 112; so auch Welsh 2010) In der Herausbildung von Normen sind also offensichtlich nicht nur lineare, sondern auch widerläufige Tendenzen am Werk, die in manchmal dialektischer Weise wirken. Luck betont, dass die Charakterisierung des Gipfels 2005 als ,tipping point" noch nicht die Frage beantworte, ob sich die Norm an diesem Punkt vor oder vielleicht sogar zurück neige (Luck 2010: 117). Die festgestellten Abwehrreaktionen einzelner Mitgliedstaaten auf die eigentlich angenommene Norm illustrieren diesen Punkt, der in jüngeren Arbeiten zum Normenwandel im Allgemeinen explizit thematisiert wird (Wiener 2009). Betrachtet man die Bezugnahme auf R2P in Resolution 1973 durch den Sicherheitsrat als weiteren ,tipping point“", ist auch hier festzustellen, dass sich erst zeigen wird, in welche Richtung sich die Norm mit dem Versuch einer Umsetzung von R2P neigen wird. ${ }^{5}$ Fest steht jedoch: Eine Nicht-Bezugnahme auf die R2P, eine Übergehung des Konzepts angesichts der Notlage, hätte sehr wahrscheinlich dazu geführt, dass diese in den Ruinen eines gestürmten Bengasi gleich mit hätte begraben

4 Eine nähere Auseinandersetzung mit der deutschen Positionierung kann an dieser Stelle nicht geleistet werden. Vergleiche dazu etwa Müller 2011. Die Irritation über die deutsche Enthaltung (erst die fünfte bei über dreihundert Abstimmungen in allen Jahren [west-]deutscher Präsenz im Sicherheitsrat) fällt auch deshalb so deutlich aus, weil diese nicht mit entsprechender diplomatischer Einbettung vorgenommen wurde und - anders als bei den weiteren Enthaltungsstimmen Chinas, Russlands, Indiens und Brasiliens - eben nicht von bekannten Skeptikern der R2P stammte. Vergleiche auch Fröhlich/Langehenke 2011.

5 Während sich der eher im POC-Kontext stehende Waffeneinsatz in der Elfenbeinküste, beschlossen kurze Zeit nach der Resolution zu Libyen (UN Doc. SR/Res/1975 vom 30.3.2011), als Bestätigung eines Trends darstellte, zeigten schon kurz später die Schwierigkeiten im Fall Syrien (vergleiche Kimball 2011), dass die konkrete Art und Weise der Durchsetzung von R2P immer von einer ganzen Reihe politischer, militärischer und ethischer Faktoren abhängen wird, die die Norm zugleich dem Vorwurf der Selektivität aussetzen. R2P verändert den Blick auf diese Faktoren, setzt diese jedoch nicht außer Kraft und hat (zumindest in der ICISS-Konzeption) durch das Kriterium der ,reasonable prospects" den notwendigen Abgleich von Erfolgsaussichten grundsätzlich mit einbezogen. 
werden können (Thakur 2011). Diese Einsicht verweist auf die praktisch-politischen Konsequenzen des Falls Libyen für die Norm der Schutzverantwortung.

Die Umsetzung der Operation „Odyssee Dawn“bzw. ihrer Nachfolgerin „Unified Protector" deutet auf eine erhebliche Lücke in der operativen Kapazität und militärischen Doktrin zum effektiven Schutz von Zivilisten hin (vgl. Holt/Berkman 2006). Diese Lücke zu verringern ist ebenfalls Teil und Aufgabe der weiteren Herausbildung einer ,,responsibility to protect“. Zu klären sind dabei insbesondere folgende Fragen: Wie lässt sich nach der Etablierung einer Flugverbotszone deren defensiver Charakter bewahren? Hier wird nochmals neu über das in Bosnien nicht umgesetzte Konzept von „Schutzzonen“ nachzudenken sein. Wie und in welchem Ausmaß lassen sich faktische Verschiebungen der Kräfteverhältnisse zwischen Bürgerkriegsparteien durch einen internationalen Einsatz legitimieren? Wenn die internationale Verantwortung zum Schutz gerade in jenen Fällen gilt, in denen sich Regierungen gegen ihr eigenes Volk wenden und Verbrechen bis hin zum Genozid verüben - bedeutet dies dann nicht zugleich, dass ein Regimewechsel unausweichlich ist? Welche Folgen jedoch dieser wiederum haben würde, ist angesichts der Strukturen in Libyen (Wehrey 2011) nur schwer vorherzusagen. Eine optimistische Variante würde die Konsolidierung eines mittelfristig versöhnten, politische und wirtschaftliche Teilhabe gewährleistenden Staates wünschen - nicht ausgeschlossen werden können jedoch auch die von Doyle skizzierten negativen Varianten einer Perpetuierung des Bürgerkrieges, eines internationalen Protektorates oder einer Tyrannei der Sieger, die sich an der unterlegenen Partei rächen (Doyle 2011). Bei aller Unwägbarkeit dieser Fragen bleibt der grundlegende Impuls von R2P aber auch die Nicht-Hinnehmbarkeit von Völkermord, Kriegsverbrechen, ethnischer Säuberung und Verbrechen gegen die Menschlichkeit. Gesucht sind nicht nur die Gründe zur Ablehnung oder Kritik an notwendigerweise riskanten Einmischungen von außen, sondern auch alternative Handlungsmöglichkeiten angesichts unmittelbar bevorstehender Massaker, in denen mittelfristige Sanktionen schlichtweg wirkungslos sind.

Jeglicher Rückgriff auf militärische Mittel ist zunächst auch eine Bankrotterklärung der Politik. Jegliche Militäraktion unterliegt einer zumindest teilweisen Unkontrollierbarkeit. Jeglicher Einsatz von Gewalt bringt Leid - nicht nur über direkt Schuldige, sondern (bei aller „Präzision“ der eingesetzten Waffen) auch Leid und Tod über Unschuldige. Wie man diese Opfer eines Einschreitens gegen die Opfer eines Nicht-Einschreitens abwägen kann, ist eine immer neu zu bewältigende Herausforderung, bei der es keine leichten Antworten gibt. Genau diese Erfahrung stand ja am Beginn der Formulierung der R2P und aus ihr erwuchs der mit dieser Formel verbundene Anspruch einer Strategie zur Verhinderung von Katastrophen wie in 
Ruanda oder Srebrenica. Das Spannungsverhältnis zwischen Souveränität und Intervention, Schutz und Gefährdung, Gewaltlegitimation und Gewaltlimitation abzuwägen, ist Aufgabe von Politik, die die theoretische wie praktische Relevanz der Kraft von Ideen, von Bedingtheiten und Möglichkeiten des Normenwandels nicht unterschätzen sollte. Dies jedenfalls scheint auch der eingangs zitierte Goethe selbst im Pulverdampf von Valmy nicht getan zu haben.

\section{Literatur}

Ban, Ki-moon, 2011: Statement on Libya vom 17.3.2011, http://www.un.org/apps/ news/infocus/sgspeeches.

Bellamy, Alex J., 2009: Responsibility to Protect. The Global Effort to End Mass Atrocities, Cambridge.

Bennis, Phyllis, 2011: Libya and the limits to the R2P, in: New Routes 16 (2), 36-39.

Chesterman, Simon, 2001: Just War or Just Peace? Humanitarian Intervention and International Law, Oxford u. a.

Doyle, Michael, 2011: The Folly of Protection. Is Intervention Against Qaddafi's Regime Legal and Legitimate?, in: Foreign Affairs Report: Libya in Crisis, $\mathrm{http}$ //www.foreignaffairs.com/articles/67666/michael-w-doyle/the-folly-ofprotection?page=show (Stand: 14.6.2011).

Euronews, 2011: Saif al-Islam Gaddafi: "In 48 Stunden ist alles vorbei." Interview vom 16.3.2011, http://de.euronews.net/2011/03/16/saif-al-islam-gaddafi-in-48stunden-ist-alles-vorbei-/ (Stand: 14.6.2011).

Evans, Gareth, 2009: The Responsibility to Protect. Ending Mass Atrocity Crimes Once and For All, Washington.

FAZ, 2011: „Wir wollen nicht warten, bis sich die Leichen türmen.“ Interview mit Edward C. Luck, in: Frankfurter Allgemeine Zeitung, 24.3.2011, 6.

Finnemore, Martha/Sikkink, Kathryn, 1998: International Norm Dynamics and Political Change, in: International Organization 52 (4), 887-917.

Fixdal, Mona/Smith, Dan, 1998: Humanitarian Intervention and Just War, in: Mershon International Studies Review 42 (2), 283-312.

Fröhlich, Manuel, 2004 a: Zwischen Friedensformel und Kriegsgrund: Der Kampf um Souveränität, in: Osnabrücker Jahrbuch Frieden und Wissenschaft 11. Außenpolitik zwischen Krieg und Frieden, Göttingen, 157-165.

Fröhlich, Manuel, 2004 b: Die Vereinten Nationen am Scheideweg, in: Bundesakademie für Sicherheitspolitik (Hrsg.), Sicherheitspolitik in neuen Dimensionen. Ergänzungsband I, Hamburg u. a., 427-448. 
Fröhlich, Manuel, 2005: Lesarten der Souveränität, in: Neue politische Literatur 50 (1), 19-42.

Fröhlich, Manuel, 2006: „Responsibility to Protect“ - Zur Herausbildung einer neuen Norm der Friedenssicherung, in: Varwick, Johannes/Zimmermann, Andreas (Hrsg.), Die Reform der Vereinten Nationen - Bilanz und Perspektiven, Berlin, 167-186.

Fröhlich, Manuel/Langehenke, Christian, 2011: Enthaltsamkeit bei Enthaltungen. Das deutsche Abstimmungsverhalten im UNO-Sicherheitsrat, in: Vereinte Nationen 59 (4) (i.E.).

Goethe, Johann Wolfgang, 1988: Kampagne in Frankreich 1792, in: Goethes Werke in zwölf Bänden. Zehnter Band, Berlin/Weimar, 361-543.

Holt, Viktoria K./Berkman, Tobias C., 2006: The Impossible Mandate? Military Preparedness, the Responsibility to Protect and Modern Peace Operations, Washington.

Human Security Center, 2005: Human Security Report 2005. War and Peace in the $21^{\text {st }}$ Century, New York u. a.

ICISS (International Commission on Intervention and State Sovereignty), 2001: The Responsibility to Protect. Report of the International Commission on Intervention and State Sovereignty, December 2001, http://iciss.ca/report-en.asp (Stand: 7.6.2011).

Kimball, Spencer, 2011: UN takes proactive role in world politics after Arab Spring, http://www.dw-world.de/dw/article/0,,15067840,00.html vom 15.5.2011 (Stand: 16.6.2011).

Kreuter-Kirchhof, Charlotte, 2010: Völkerrechtliche Schutzverantwortung bei elementaren Menschenrechtsverletzungen. Die Responsibility to Protect als Verantwortungsstruktur, in: Archiv des Völkerrechts 48 (3), 338-382.

Luck, Edward, 2010: Building a Norm: The Responsibility to Protect Experience, in: Rotberg, Robert I. (Hrsg.), Mass Atrocity Crimes. Preventing Future Outrages, Cambridge u. a., 108-127.

Müller, Harald, 2011: Ein Desaster. Deutschland und der Fall Libyen. Wie sich Deutschland moralisch und außenpolitisch in die Isolation manövrierte, HSFK Standpunkt 2/2011, Frankfurt a. M.

Mueller, John, 2011: The Iraq Syndrome Revisited: U.S. Intervention, From Kosovo to Libya, in: Foreign Affairs Report: Libya in Crisis, http://www.foreignaffairs.com/articles/67681/john-mueller/the-iraq-syndrome-revisited?page=show (Stand: 14.6.2011).

Obama, Barack/Cameron, David/Sarkozy, Nicolas, 2011: Libya's Pathway to Peace, New York Times, 14.4.2011. 
O'Hanlon, Michael, 2011: Winning Ugly in Libya: What the United States Should Learn From Its War in Kosovo, in: Foreign Affairs Report: Libya in Crisis, http:// www.foreignaffairs.com/articles/67684/michael-ohanlon/winning-ugly-inlibya?page=show (Stand: 14.6.2011).

Sinjen, Svenja, 2011: Der Preis der Freiheit. Fall Libyen: Was wir neu denken müssen, in: Internationale Politik 66 (3), 78-83.

Strauss, Ekkehard, 2009: The Emperor's New Clothes? The United Nations and the Implementation of the Responsibility to Protect, Baden-Baden.

Thakur, Ramesh, 2011: UN breathes Life into Responsibility to Protect, Toronto Star, 21.3.2011.

The Editors, 2011: What Qaddafi Said. Excerpts from Libyan Leader Muammar alQaddafi's Last Televised Address, in: Foreign Affairs Report: Libya in Crisis, http://www.foreignaffairs.com/articles/67878/the-editors/what-qaddafi-said? page $=$ show (Stand: 14.6.2011).

Verlage, Christopher, 2009: Responsibility to Protect. Ein neuer Ansatz im Völkerrecht zur Verhinderung von Völkermord, Kriegsverbrechen und Verbrechen gegen die Menschlichkeit, Tübingen.

Wehrey, Frederic, 2011: Libya's Terra Incognita. Who and What Will Follow Qaddafi?, in: Foreign Affairs Report: Libya in Crisis, http://www.foreignaffairs.com/articles/67551/frederic-wehrey/libyas-terra-incognita?page=show (Stand: 14.6.2011).

Welsh, Jennifer, 2010: Implementing the Responsibility to Protect: Where Expectations Meet Reality, in: Ethics \& International Affairs 24 (4), 415-430.

Wiener, Antje, 2009: Enacting Meaning-in-Use: Qualitative Research on Norms and International Relations, in: Review of International Studies 35 (1), 175-193. 
Korrespondenzanschrift:

Prof. Dr. Manuel Fröhlich

Friedrich-Schiller-Universität Jena

Fakultät für Sozial- und Verhaltenswissenschaften

Professur für Internationale Organisationen und Globalisierung

Carl-Zeiß-Straße 3

D-07743 Jena

E-Mail: manuel.froehlich@uni-jena.de 